\title{
How is open solar magnetic flux lost over the solar cycle?
}

\section{Article}

Published Version

Owens, M. J., Crooker, N. U. and Lockwood, M. (2011) How is open solar magnetic flux lost over the solar cycle? Journal of Geophysical Research - Space Physics, 116. A04111. ISSN 0148-0227 doi: https://doi.org/10.1029/2010JA016039 Available at https://centaur.reading.ac.uk/20350/

It is advisable to refer to the publisher's version if you intend to cite from the work. See Guidance on citing.

To link to this article DOI: http://dx.doi.org/10.1029/2010JA016039

Publisher: American Geophysical Union

All outputs in CentAUR are protected by Intellectual Property Rights law, including copyright law. Copyright and IPR is retained by the creators or other copyright holders. Terms and conditions for use of this material are defined in the End User Agreement.

\section{www.reading.ac.uk/centaur}

\section{CentAUR}

Central Archive at the University of Reading

Reading's research outputs online 


\title{
How is open solar magnetic flux lost over the solar cycle?
}

\author{
M. J. Owens, ${ }^{1,2}$ N. U. Crooker, ${ }^{3}$ and M. Lockwood ${ }^{1}$ \\ Received 20 August 2010; revised 19 January 2011; accepted 8 February 2011; published 28 April 2011.
}

[1] The Sun's open magnetic field, magnetic flux dragged out into the heliosphere by the solar wind, varies by approximately a factor of 2 over the solar cycle. We consider the evolution of open solar flux in terms of a source and loss term. Open solar flux creation is likely to proceed at a rate dependent on the rate of photospheric flux emergence, which can be roughly parameterized by sunspot number or coronal mass ejection rate, when available. The open solar flux loss term is more difficult to relate to an observable parameter. The supersonic nature of the solar wind means open solar flux can only be removed by near-Sun magnetic reconnection between open solar magnetic field lines, be they open or closed heliospheric field lines. In this study we reconstruct open solar flux over the last three solar cycles and demonstrate that the loss term may be related to the degree to which the heliospheric current sheet (HCS) is warped, i.e., locally tilted from the solar rotation direction. This can account for both the large dip in open solar flux at the time of sunspot maximum as well as the asymmetry in open solar flux during the rising and declining phases of the solar cycle. The observed cycle-to-cycle variability is also well matched. Following Sheeley et al. (2001), we attribute modulation of open solar flux by the degree of warp of the HCS to the rate at which opposite polarity open solar flux is brought together by differential rotation.

Citation: Owens, M. J., N. U. Crooker, and M. Lockwood (2011), How is open solar magnetic flux lost over the solar cycle?, J. Geophys. Res., 116, A04111, doi:10.1029/2010JA016039.

\section{Introduction}

[2] A component of the magnetic flux which threads the photosphere is carried out to the heliopause by the solar wind. Flux threading the heliopause is one topological definition of "open" solar flux, but this cannot be measured. Other definitions are of flux threading an arbitrarily chosen surface closer to the Sun, and are measurable. However defined, open solar flux partially shields Earth from galactic cosmic rays (GCRs) [Cane et al., 1999]. GCR records in ice cores, ocean sediments and tree trunks provide a means of inferring the prehistoric heliospheric magnetic field, which in turn can be used to reconstruct the photospheric field and hence solar irradiance variations crucial for long-term climate modeling [e.g., Lockwood, 2006 and references therein]. A vital link in this chain is the relation between photospheric and heliospheric magnetic flux, in particular the physical processes by which the Sun's open flux varies over solar cycle and secular timescales.

\footnotetext{
${ }^{1}$ Space Environment Physics Group, Department of Meteorology, University of Reading, Reading, UK.

${ }^{2}$ Space and Atmospheric Physics, Blackett Laboratory, Imperial College London, London, UK. USA.

${ }^{3}$ Center for Space Physics, Boston University, Boston, Massachusetts,

Copyright 2011 by the American Geophysical Union. 0148-0227/11/2010JA016039
}

[3] At the photosphere, the magnetic field can be remotely observed by spectroscopic techniques, allowing a complete map of the line-of-sight photospheric magnetic field to be constructed once per solar rotation $(\sim 27$ days, one Carrington rotation, from the vantage point of Earth). Total (unsigned) photospheric magnetic flux shows a strong solar cycle variation, qualitatively similar to the sunspot variation [e.g., Arge et al., 2002]. Most of this flux, however, does not contribute to the heliosphere: A loop must have an apex above the solar wind acceleration height in order to be dragged out into the heliosphere. It is not possible to estimate what fraction of photospheric flux is open to the heliosphere on the basis of photospheric observations alone.

[4] The potential field source surface (PFSS) model [Schatten et al., 1969; Altschuler and Newkirk, 1969] has been particularly successful in extrapolating from photospheric observations to global coronal structure. The "potential field" assumption is equivalent to zero current density in the corona, meaning PFSS solutions approximate the lowest-energy state of the corona for a given photospheric boundary condition. The outer boundary of the PFSS is the "source surface" where the field is assumed to be radial, typically placed at $2.5 R_{S}$ [e.g., Arge and Pizzo, 2000]. Open flux is then frequently defined as any magnetic loop threading this surface. At solar minimum, the foot points of open solar field lines largely map to the polar regions and are strongly associated with the dark regions in soft X-ray and EUV images known as coronal holes. PFSS estimates of open flux show a solar cycle variation in gen- 


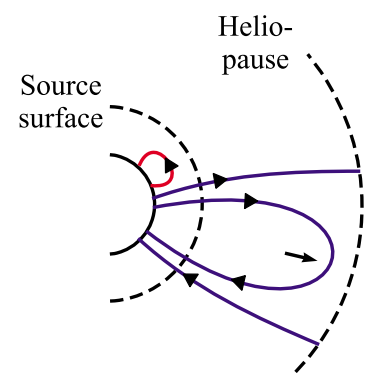

\section{Open / closed solar flux}

Figure 1. "Open solar flux" is defined as field lines which thread the source surface, regardless of whether they close in the heliosphere. "Open heliospheric flux" is then a subset of open solar flux, being field lines which go on to thread the heliopause.

eral agreement with heliospheric observations [Wang and Sheeley, 1995, 2003; Lockwood et al., 2009b] (though see also Riley [2007] for a conflicting view). An alternative to the PFSS model is the when a current sheet source surface model [Schüssler and Baumann, 2006].

[5] In the heliosphere, in situ spacecraft measurements enable precise, high-cadence observations of the vector magnetic field, but at a single point in space, making estimation of global parameters problematic. An observed latitude invariance in $B_{R}$, the radial component of the heliospheric magnetic field [Smith and Balogh, 2003; Lockwood et al., 2004; Owens et al., 2008a], however, means that $\left|B_{R}\right|$ measured at any point in the heliosphere is representative of the total open solar flux threading a heliocentric sphere to a high degree of accuracy [Lockwood and Owens, 2009]. Measurements at $1 \mathrm{AU}$ can be used to estimate the source surface flux by correcting for kinematic effects which can amplify $\left|B_{R}\right|$ during the solar wind transit from the Sun to the observer [Lockwood et al., 2009a, 2009b]. This measure of open solar flux exhibits a solar cycle variation, approximately doubling from solar minimum to solar maximum. There is also cycle-to-cycle variability, with the current solar minimum exhibiting the lowest values since observations began.

[6] While the solar cycle variation in open solar flux is well characterized, determination of the processes responsible is difficult. In situ observations are limited to a single point in space and result in ambiguity between spatial and temporal evolution. PFSS extrapolations, on the other hand, are global, but present a series of snapshots rather than a continuously evolving solution and thus cannot directly reveal the dynamics of open flux variation [see, however, Luhmann et al., 1998, 1999; Wang and Sheeley, 2003; Yeates et al., 2010]. This study aims to bring together these data sets to better understand how the corona dynamically adds to and removes magnetic flux from the heliosphere. Section 2 attempts to describe the most relevant work to date, section 3 summarizes the available observations and section 4 compares different models of open solar flux with observations. The implications of our findings as discussed in section 5 .

\section{Background}

\subsection{Defining Open Flux}

[7] Without violation of Maxwell's laws, all magnetic flux must ultimately form closed loops, but it is nevertheless useful in many situations to talk in terms of "open" flux, meaning field lines which reach some boundary before they close. In the solar literature, the source surface is often used as the open/closed boundary [e.g., Wang and Sheeley, 1995; Arge and Pizzo, 2000]. In the heliosphere, where all field lines are open using the source surface definition, there are observational signatures of magnetic loops with both foot points in the solar atmosphere (and hence termed "closed") and so "open flux" is often defined as flux which reaches the heliopause, where it presumably reconnects with the interstellar magnetic field.

[8] Figure 1 shows the definitions used in this study. Figure 1 (left) shows "open solar flux," $\Phi_{O S}$, in blue. It is defined as field lines which thread the source surface, regardless of whether they close in the heliosphere. Closed coronal loops, shown in red, do not contribute to open solar flux. Figure 1 (right) shows "open heliospheric flux" in blue. It is a subset of open solar flux, being field lines which go on to thread the heliopause. Closed heliospheric loops, shown in red, also contribute to open solar flux. They are usually associated with coronal mass ejections, as discussed below.

[9] The supersonic nature of the solar wind means magnetic flux can only be transported radially outward through the source surface. Magnetic reconnection above the source surface is unable to alter either the total open solar flux nor its configuration below the source surface. The only way to increase open solar flux is by transporting a closed loop past the source surface. Similarly, the only way to reduce open solar flux is through two open solar field lines reconnecting below the source surface. We refer to this generic $\Phi_{O S}$ loss process as "pinching." It can take two forms: "Disconnection" occurs when both reconnecting open solar field lines form open heliospheric flux, resulting in the formation of heliospheric flux with no connection to the Sun [Wang et al., 1999]. For completeness, we note the possibility of disconnection occurring by reconnection between field lines which are part of the same closed heliospheric loop, though this seems unlikely in three dimensions and is not shown in Figure 1. If either of the open solar field lines is part of a closed heliospheric loop, however, "interchange reconnection" instead occurs and no flux is actually disconnected from the Sun [Crooker et al., 2002]. Figure 2 shows these processes schematically. Note that both forms of pinching, disconnection and interchange reconnection, lead to the same reduction in open solar flux.

\subsection{Observational Constraints}

[10] It is possible to describe the variation in open solar flux, $\Phi_{O S}$, in terms of a source term $S$ and a loss term $L$ [Solanki et al., 2000]:

$$
\frac{d \Phi_{O S}(t)}{d t}=S-L
$$



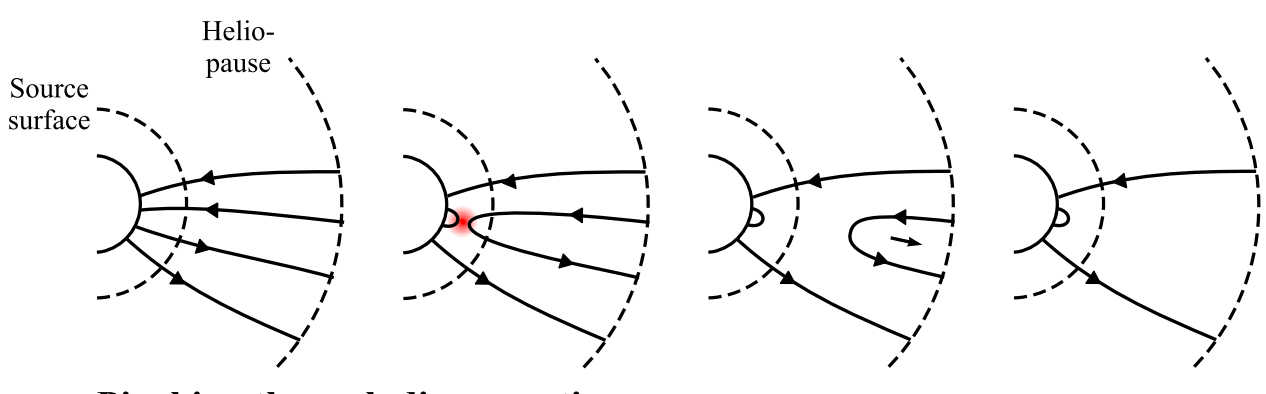

Pinching through disconnection
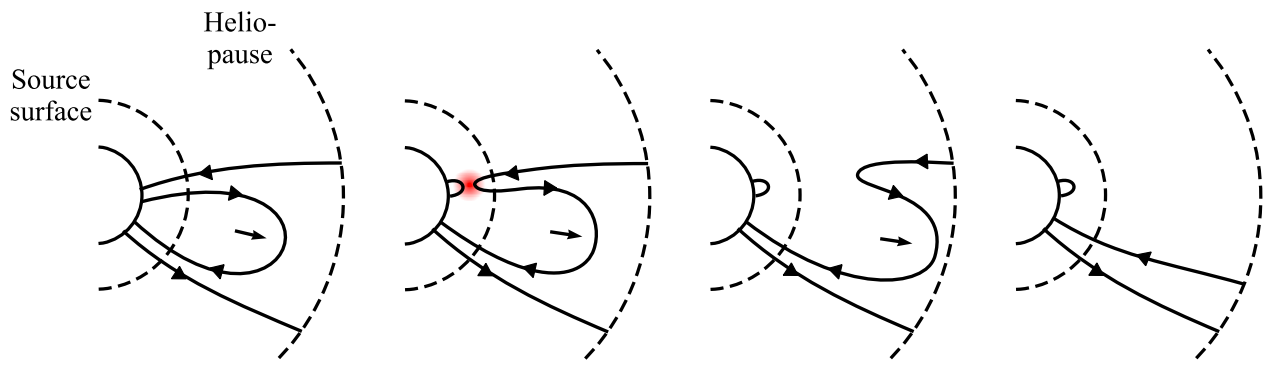

\section{Pinching through interchange reconnection}

Figure 2. Cartoons of "pinching", reconnection between open solar field lines which occurs below the source surface, leading to loss of open solar flux. (top) The reconnecting field lines are both open heliospheric field lines, leading to a heliospheric flux completely disconnected from the Sun. (bottom) The "interchange reconnection," which involves open and closed heliospheric field lines. It does not produce disconnected field lines in the heliosphere. Note that both processes lead to the same reduction in open solar flux.

Based upon the above arguments/definitions, $S$ must describe the rate at which closed coronal loops are added to the heliosphere, whereas $L$ must describe the rate at which pinching occurs below source surface.

[11] Closed heliospheric loops can be identified by the presence of counterstreaming suprathermal electrons (CSEs) [Gosling et al., 1987]. This signature may only be present for newly added heliospheric loops, as the apex of a loop will continue to move toward the heliopause at the solar wind speed and eventually the loop will become long enough that the CSE signature is lost by pitch angle scattering [Owens and Crooker, 2007; Owens et al., 2008b]. CSEs are highly correlated with the passage of coronal mass ejections [Gosling et al., 1987], meaning coronal mass ejections (CMEs) are undoubtedly a source of new open solar flux [Low, 2001].

[12] Ambient (i.e., non-CME associated) coronal loops which rise through the source surface to become heliospheric loops will also add to the net open solar flux. CSEs are thought to arise from the high temperatures of the corona and not from the CME eruption mechanism, meaning they should be present on all newly emerging heliospheric loops. The lack of significant CSEs in the absence of other CME signatures suggests rising ambient loops are a minor contribution to new open solar flux.

[13] There are few direct, observational constraints on the loss rate of open solar flux. McComas et al. [1992] argued that pinching cannot proceed primarily by disconnection, as this would produce suprathermal electron dropouts at a rate much higher than observed. Owens and Crooker [2007], however, demonstrated that the paucity of electron dropouts is not sufficient to discount disconnection. The mixture of open and closed heliospheric flux within CMEs, however, supports the idea that interchange reconnection, rather than disconnection, removes a significant amount of open solar flux [Crooker et al., 2008].

[14] Connick et al. [2010] separated in situ heliospheric magnetic field observations into components parallel and perpendicular to the Parker spiral, which they interpreted as measures of open flux and CME flux, respectively. From the time variations of these components over the recent solar cycle, they argued that the rate of flux loss was likely to be constant.

[15] Sheeley and Wang [2001] showed an association between coronal inflows, assumed to be a signature of pinching between the Sun and the reconnection point [Wang et al., 1999], and the locations where the heliospheric current sheet (HCS) was inclined to the solar rotation direction. This local HCS inclination can be the result of either a global tilt or small-scale HCS warping. Sheeley and Wang [2001] suggested that open solar flux either side of the current sheet may be forced together by differential rotation, leading to pinching. This process is similar to the open solar flux transport required at coronal hole boundaries in order for them to rigidly rotate despite the differential rotation of the photospheric magnetic field [Nash et al., 1988; Wang and Sheeley, 2004]. It may also provide a means of closing the large-scale circulation cells of open solar flux when the dipole axis is tilted with respect to the rotation axis [Fisk et al., 1999]. 


\subsection{Current Models of Open Solar Flux}

[16] On this basis of these observations, Owens and Crooker [2006] modeled the solar cycle 23 variation in heliospheric magnetic field strength (directly related to the total solar open flux) assuming all new closed loops added to the heliosphere are carried by CMEs. Their open solar flux source term was $S=\phi_{C M E} f_{C M E}$, where $f_{C M E}$ is the Large Angle and Spectrometric Coronagraph- (LASCO) derived CME rate [St. Cyr et al., 2000; Yashiro et al., 2004; Gopalswamy et al., 2008] and $\phi_{C M E}$ is the observational estimate of the typical magnetic flux content of a CME [Lynch et al., 2006]. This scheme therefore does not account for flux added to the heliosphere by transients too small to be classed as CMEs, or by rising ambient coronal loops. Owens and Crooker [2006] assume that all pinching takes place by interchange reconnection with closed heliospheric flux carried by CMEs (Figure 2, bottom). $L$ is proportional to the instantaneous amount of closed heliospheric flux, with a timescale for flux removal as a free parameter of the model. They found reasonable agreement between the model and observed variation in heliospheric field strength over solar cycle 23 . Note that as the CME rate tends toward zero, so must the open solar flux loss term. Therefore this model allows, though does not necessarily require, the existence of a non-zero "floor" in the open solar flux.

[17] Schwadron et al. [2010] also assume CMEs to be the sole source of open solar flux, though they use a purely sinusoidal variation for the CME rate, enabling analysis to be extended to the pre-LASCO era. They assume two loss terms: One proportional to the instantaneous closed heliospheric flux, as per Owens and Crooker [2006], and a very slowly changing loss term associated with disconnection of open heliospheric flux.

[18] Vieira and Solanki [2010] and Krivova et al. [2007] divide open solar flux into active region and ephemeral contributions. The active region source term is assumed to be proportional to sunspot number $(R)$, whereas the ephemeral source term is characterized by the length, amplitude and timing of multiple $R$ cycles. The use of $R$ as a proxy for heliospheric magnetic flux injection allows analysis of the pre-coronagraph era. As CME rates are highly correlated with sunspot number (SSN) (see also Figure 3) [Webb and Howard, 1994], the open solar flux source terms for the various open solar flux models are very similar. Both Vieira and Solanki [2010] source terms have a timescale over which their contribution to open solar flux decays, with the ephemeral term decaying significantly slower than the active region term. This means that active region flux determines much of the solar cycle variation, with the ephemeral term describing much longer cycle-to-cycle variations.

\section{Data}

\subsection{Summary of the Last Three Solar Cycles}

[19] Figure 3 shows a summary of the key solar/heliospheric parameters over the last three solar cycles. Figure 3 (top) shows open solar flux computed from $1 \mathrm{~h}$ OMNI near-Earth observations (available from the National Space Science Data Center http://omniweb.gsfc.nasa.gov/) of the radial heliospheric magnetic field component, $B_{R}(1 A U)$. It is assumed that the total unsigned flux threading a $1 \mathrm{AU}$ heliocentric sphere can be approximated by Carrington rotation averages of $4 \pi A U^{2}\left|B_{R}(1 A U)\right|$ [Owens et al., 2008a]. The thick line shows a three Carrington rotation averages of hourly data.

[20] Kinematic effects arising from stream-stream interactions can amplify the total unsigned flux threading heliocentric spheres at increasing distance from the Sun. The thin line in Figure 3 (top) shows the hourly 1 AU flux kinematically corrected to the source surface using the observed longitudinal solar wind structure [Lockwood et al., $2009 \mathrm{a}, 2009 \mathrm{~b}]$. We note that the reduction in $4 \pi A U^{2}$ $\left|B_{R}(1 A U)\right|$ obtained from correcting to the source surface is quantitatively similar to taking 1 day averages of $B_{R}$ before taking the magnitude. This may explain why PFSS estimates agree well with 1 day averages of $B_{R}$ [Wang and Sheeley, 1995; Lockwood et al., 2009b], but underestimate the flux computed from higher-resolution measurements of $B_{R}$ [Lockwood et al., 2009b]. Note that while the magnitude of open flux is reduced by the kinematic correction, the phasing is unchanged.

[21] Figure 3 (middle) shows Carrington rotation averages of the Greenwich sunspot number $(R)$. Overlaid in red are Carrington rotation averages of coronagraph-derived daily CME rates, processed in the same manner as Owens et al. [2008c]. They have been scaled up by a factor of 20 so they can be viewed on the same axis as $R$. For the purpose of discussion, we have highlighted times when the open solar flux increases during rising phases of the sunspot cycle in blue and open solar flux decreases during the declining phases of the sunspot cycle in red.

[22] Sunspot number and CME rate vary approximately sinusoidally over the solar cycle, though the rise to maximum is steeper than the decline to minimum. At the peak of the solar cycle, between the shaded rise and declining phases, there is a small drop in sunspot number, at least for cycles 22 and 23 [Gnevyshev, 1977; Richardson et al., 2002]. Bounding this "Gnevyshev gap," $R$ shows a slight asymmetry, with the end of the rise phase exhibiting marginally higher sunspot number than the start of the declining phase.

[23] Open solar flux also shows a solar cycle variation, with the overall envelope and phase in rough agreement with the $R$ variation. There are, however, a number of significant differences. The drop in open solar flux at the Gnevyshev gap is much more pronounced than that in sunspot number, meaning the solar cycle variation in $\Phi_{O S}$ is closer to bimodal, with broad peaks either side of the gap. The $\Phi_{O S}$ rise phase peak is smaller in amplitude than the declining phase peak, the opposite sense to the $R$ asymmetry about solar maximum. Thus if $R$ is used to quantify the open solar flux source term, any model which assumes a constant loss term, a loss proportional to the source, or any combination thereof, will not be able to reproduce certain features of the observed solar cycle variation (see also section 4).

[24] We note that CME rate does show a solar cycle variation qualitatively consistent with open solar flux, in particular the correct asymmetry about the gap, though the gap itself remains a weak feature. Changing observers and instruments over this period, however, means the CME rates require careful intercalibration [Owens et al., 2008c]. Automated $\mathrm{CME}$ detection techniques may provide a more 


\section{Cycle $21 \quad$ Cycle $22 \quad$ Cycle 23}

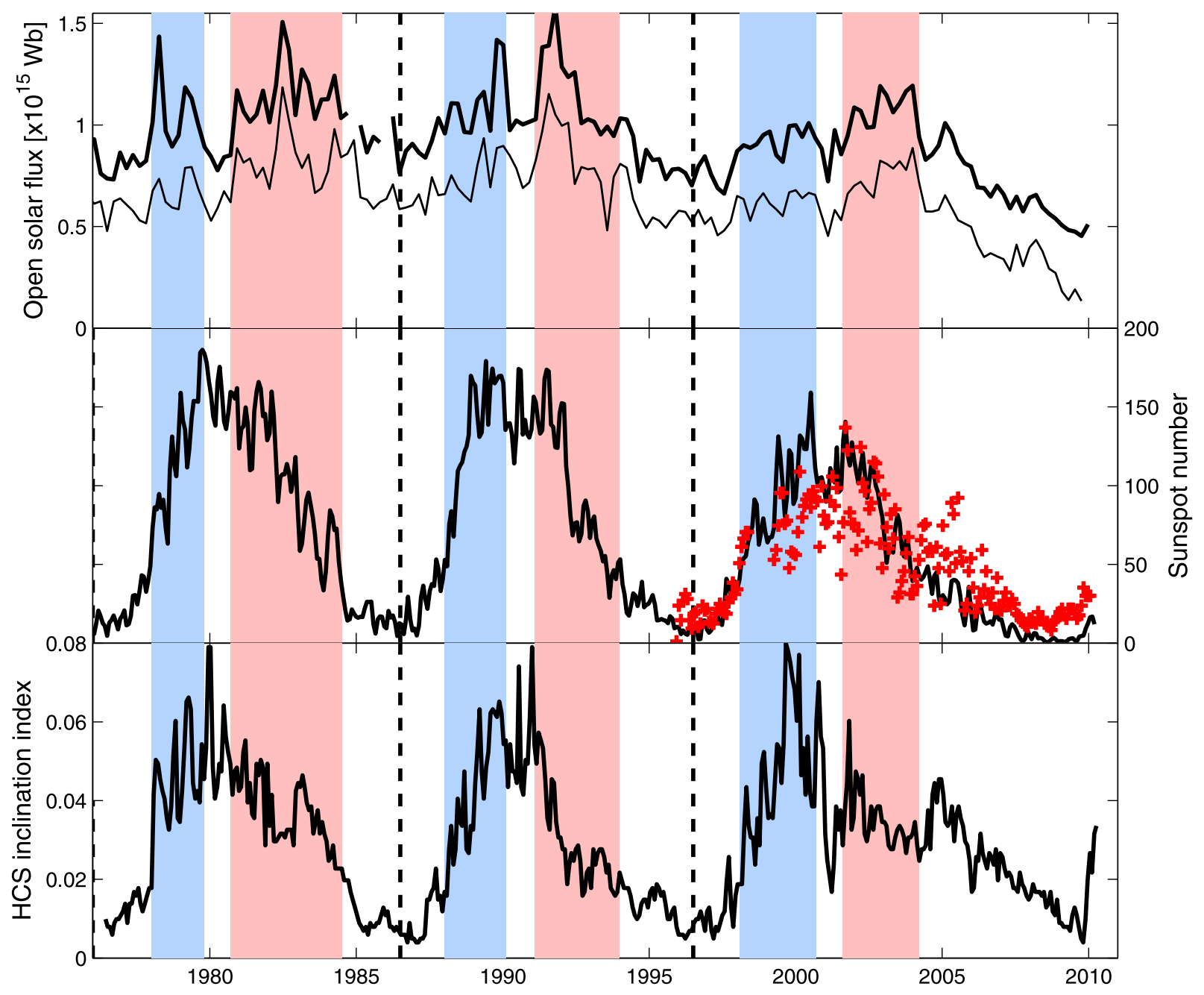

Figure 3. Key solar/heliospheric parameters over the last three solar cycles. (top) Three Carrington rotation averages of total solar open magnetic flux computed from hourly means of $1 \mathrm{AU}$ measurements of $B_{R}$. The thick line shows the measured $1 \mathrm{AU}$ value, while the thin line shows the flux kinematically corrected to the source surface. (middle) Sunspot number (black) and coronagraph-derived CME rates (red), the latter scaled up by a factor of 20. (bottom) A measure of the average local inclination of the heliospheric current sheet to the solar rotation direction, derived from PFSS solutions to the Wilcox magnetograms. The blue (red) shaded regions indicate the approximate periods of the rising (declining) phase in total solar flux referred to in the main text.

objective means of obtaining accurate $\mathrm{CME}$ rate information over the solar cycle [Olmedo et al., 2008; Robbrecht and Berghmans, 2004], though it is currently unclear how well they separate CMEs from other coronal phenomena [Yashiro et al., 2008] and are obviously still limited to the LASCO/STEREO era. In contrast, sunspot records are robust and available pre-1996.

\subsection{Local Inclination of the Heliospheric Current Sheet}

[25] In this section, we investigate the inclination of the heliospheric current sheet as a measure of the rate at which pinching occurs to reduce open solar flux. Direct observations of global HCS inclination can be assembled from in situ solar wind measurements made by the Ulysses spacecraft. Figure 4 (middle) shows the heliographic latitude of the Ulysses orbit overlaid on the sunspot number. The red and blue panels show the observed heliospheric magnetic polarity mapped back to the source surface, with blue/red as inward/outward field [Jones et al., 2003]. Complete latitude-longitude maps can be constructed during Ulysses's three fast latitude scans. The heliospheric current sheet (HCS) separating the opposite polarity regions varies significantly over the solar cycle, being approximately aligned with the rotational equator around solar minimum and with a greater inclination to the equator at solar maximum. Note also that the recent solar minimum exhibits a greater HCS inclination than the previous minimum [see also Wang and Sheeley, 2009].

[26] HCS inclination can also be inferred for each Carrington rotation from potential field source surface (PFSS) extrapolations of the observed photospheric magnetic field. 


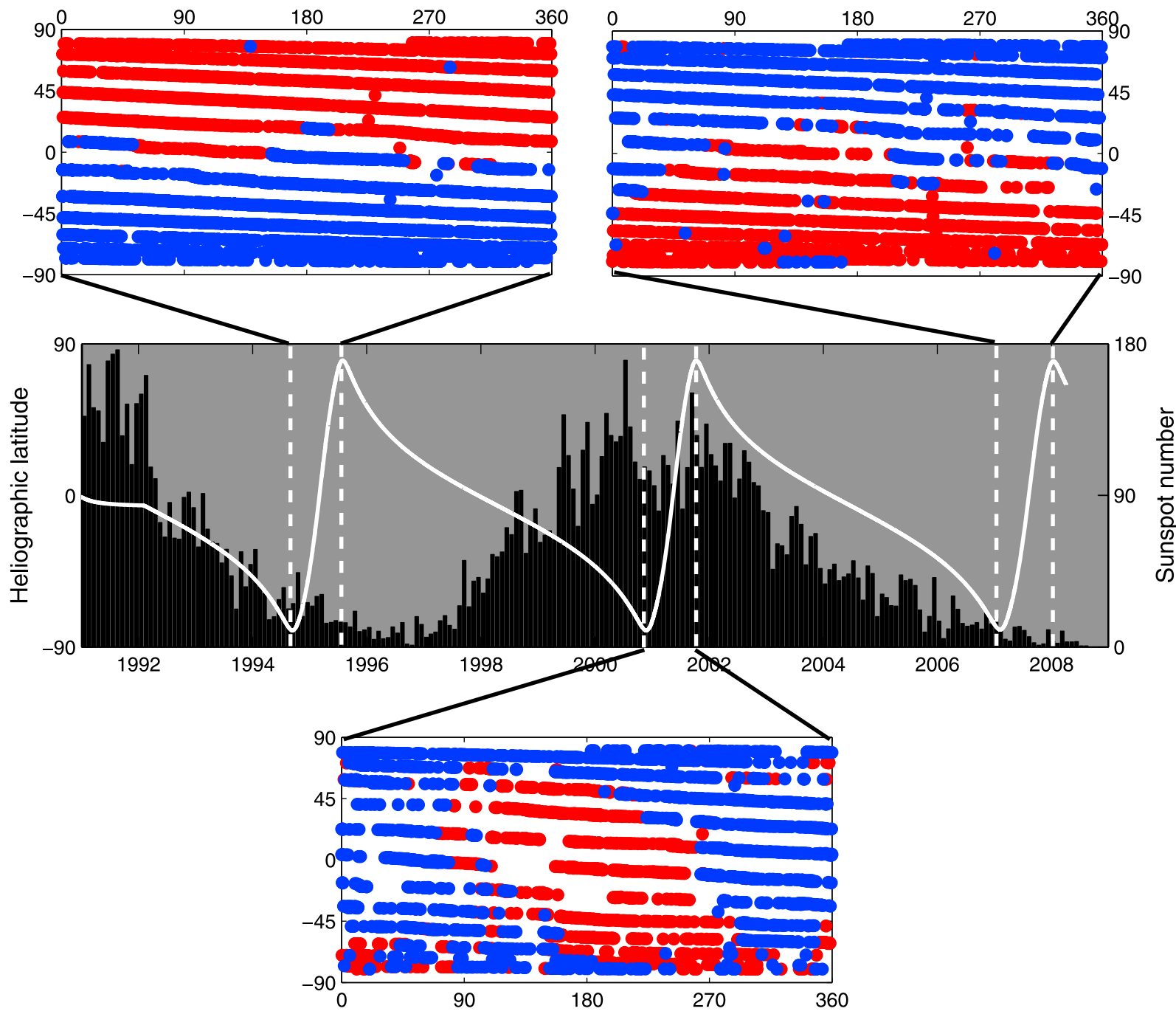

Figure 4. A summary of the observations made by Ulysses. (middle) The heliographic latitude of the spacecraft overlaid on the sunspot number. The surrounding panels show the observed heliospheric magnetic polarity mapped back to the source surface (with blue/red as inward/outward field). Complete latitude-longitude maps can be constructed during Ulysses' three fast latitude scans. The heliospheric current sheet (HCS), separating the opposite polarity regions, varies significantly over the solar cycle, being approximately aligned with the rotational equator around solar minimum and with a greater inclination to the equator at solar maximum. Note also that the recent solar minimum exhibits a greater HCS inclination than the previous minimum.

This method generally shows good agreement with both the observed coronal holes and the large-scale magnetic sector structure observed in near-Earth space [e.g., Arge and Pizzo, 2000; Owens et al., 2005] (see also Figures 4 and 5). In this study, use Wilcox Solar Observatory (WSO) magnetograms to solve the PFSS magnetic field on a $45 \times 90$ cell grid in latitude-longitude, giving an average grid cell size of $4^{\circ}$, approximately equal to the resolution of the input data. Grid cells are equally spaced in longitude and the sine of the latitude, so as to maintain constant surface area in each cell. The HCS is assumed to correspond to the magnetic neutral line at the source surface at $2.5 R_{S}$.

[27] Figure 5 shows radial magnetic field polarity at the potential field source surface for the three Carrington rotations at the midpoints of the Ulysses fast latitude scans shown in Figure 4. There is generally a good agreement, at least on a qualitative level. As was seen in the Ulysses observations, the HCS during the solar minimum at the end of cycle 23 was more inclined to the rotation direction than it was at the end of cycle 22. As the radial photospheric magnetic field is better observed at the equator than at poles, the HCS is likely to be better reproduced at solar minimum than solar maximum, when it extends to higher latitudes, though this uncertainty is difficult to quantify. As this study is concerned with the degree of HCS inclination rather than an accurate reconstruction of the HCS position, we assume that the PFSS model provides the qualitative variation of the $\mathrm{HCS}$.

[28] To quantify the average local inclination of the HCS to the solar rotation direction, we define an index, $I_{I N C}$, as 


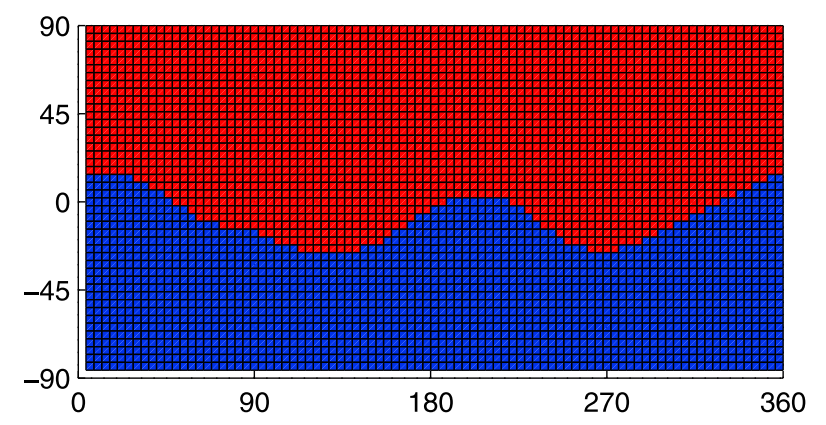

\author{
CR1891 \\ (January 1995) \\ $I_{\text {INC }}=0.0168$
}
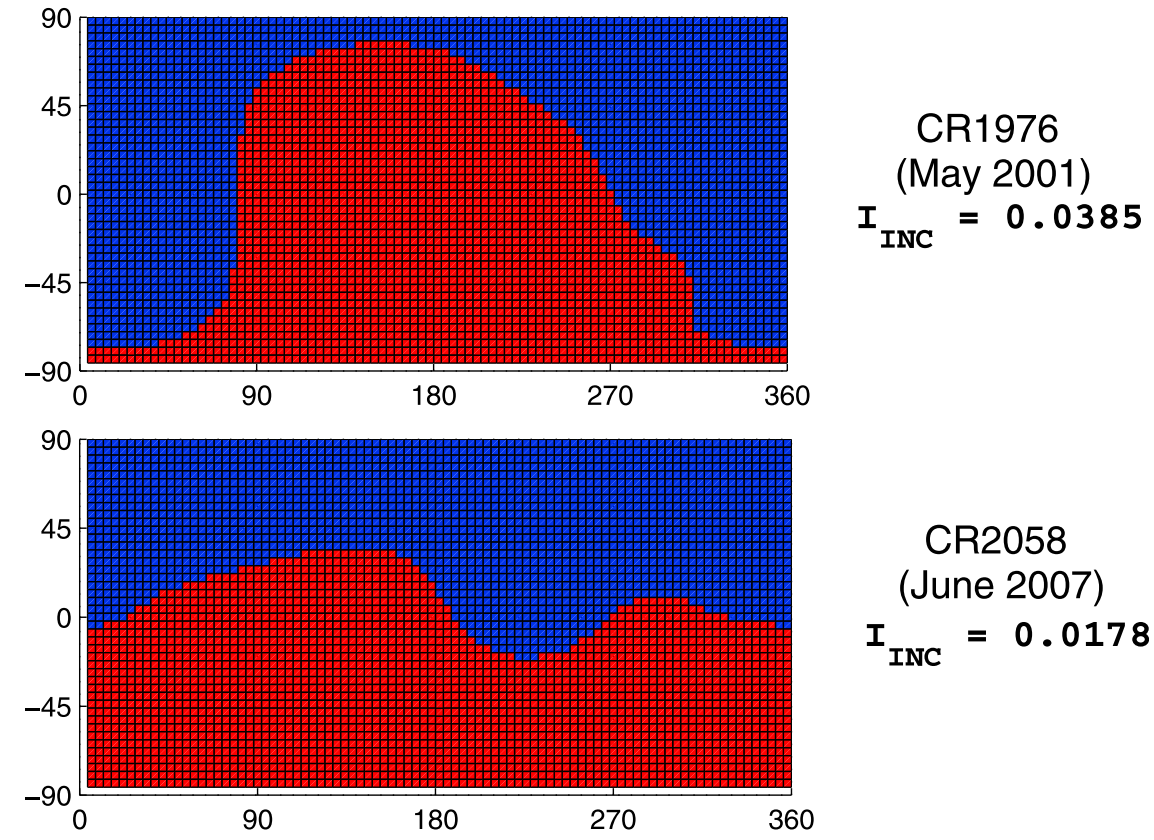

Figure 5. Maps of radial magnetic field polarity at the potential field source surface. The three Carrington rotations shown are the midpoints of the Ulysses fast latitude scans shown in Figure 4. The HCS inclination index, $I_{I N C}$, measures the fraction of the grid cells where the HCS is perpendicular to the rotation axis.

the fraction of source surface grid cells which have opposite polarity to their immediate longitudinal neighbors. Thus $I_{I N C}$ measures the fraction of the grid cells where the HCS is locally perpendicular to the rotation direction. Figure 3 (bottom) shows $I_{I N C}$ over the last three solar cycles. The variation is similar to that of the global HCS tilt angle regularly computed by WSO [Wilcox and Hundhausen, 1983], which measures the maximum latitudinal extent of the HCS. However, $I_{I N C}$ is better suited to quantifying the degree to which the HCS is also warped, meaning it can lie perpendicular to the solar rotation direction at multiple points.

[29] To first order, the HCS inclination index follows the solar cycle variation of sunspot number. Note, however, that $I_{I N C}$ is higher during the rise to maximum than in the decline to minimum. If the local HCS inclination is related to pinching [Sheeley et al., 2001] and hence the $\Phi_{O S}$ loss rate then the variation in $I_{I N C}$ may explain the asymmetry in open solar flux either side of solar maximum. The spike in $I_{I N C}$ may also be related to the drop in open solar flux at the peak of solar maximum. These ideas are quantitatively tested in section 4 .

\section{Modeling Open Solar Flux}

[30] In this section, we compare the observed $\Phi_{O S}$ variation with three models using different open solar flux loss rates. We begin by considering the coronagraph-derived CME rates, $f_{C M E}$, as a proxy for the open solar flux source term.

\subsection{CME-Dependent $\Phi_{\text {os }}$ Source Term}

[31] The black line in Figure 6 shows the variation in open solar flux, as inferred directly from hourly means of $1 \mathrm{AU}$ measurements of $B_{R}$ (i.e., without the application of the kinematic correction), with best fit model reconstructions in red using equation (1). Figure 7 shows the same results as Figure 6 , but using open solar flux inferred from $1 \mathrm{AU}$ measurements of $B_{R}$ with the application of the kinematical correction to the source surface [Lockwood et al., 2009a, $2009 \mathrm{~b}]$. The corrected and uncorrected time series show 


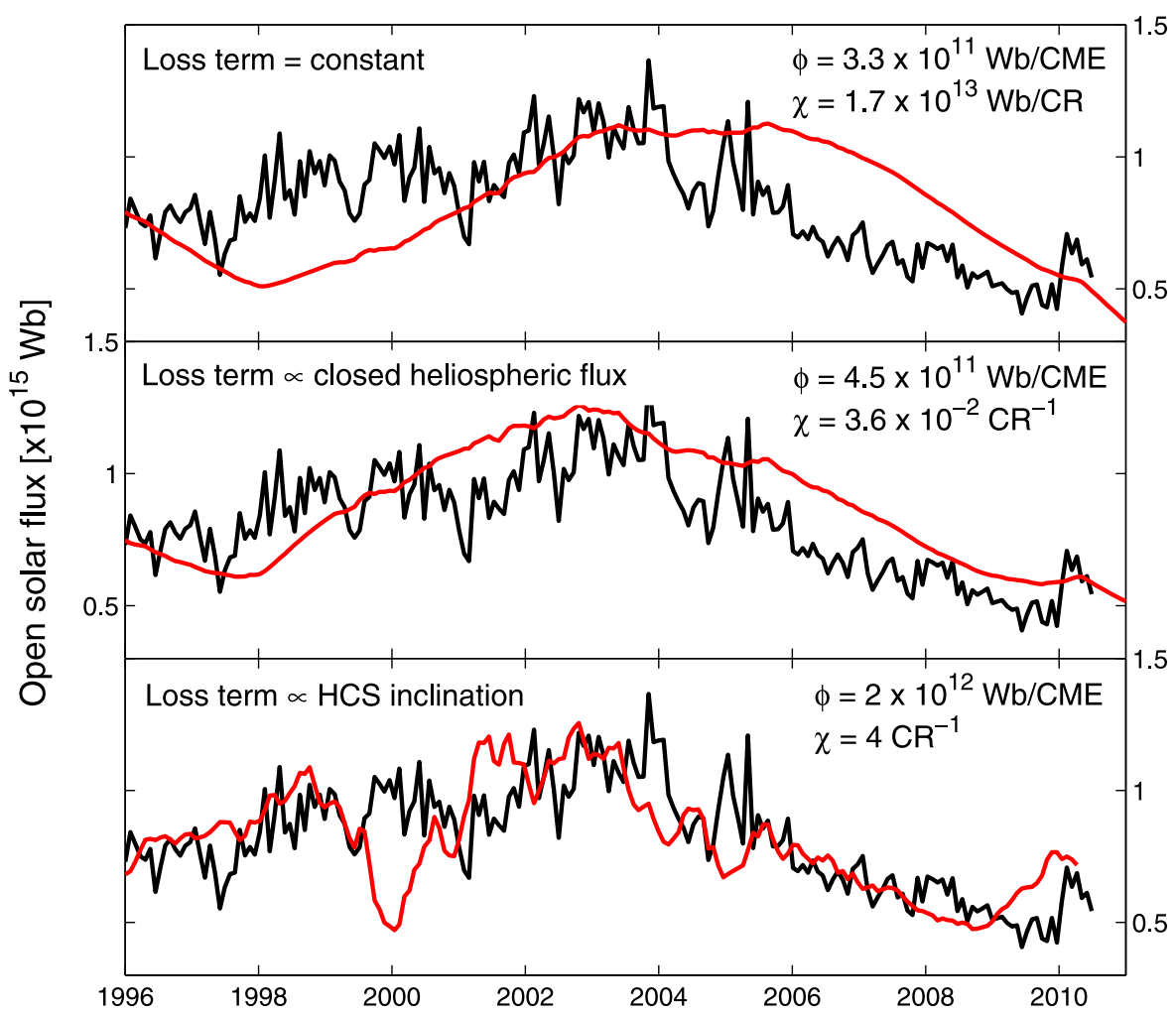

Figure 6. Modeling open solar flux using the observed CME rates. Black lines show Carrington rotation averages of the open solar flux computed from hourly means of $1 \mathrm{AU}$ observations of $B_{R}$. The red lines show model reconstructions using the coronagraph-derived CME rate as the source term. From top to bottom, the panels show open solar flux loss at a constant rate, at a rate proportional to the amount of closed heliospheric flux, and at a rate proportional the local HCS inclination.

similar variations, particularly in phasing, which is where the model is best constrained. Thus while the free parameters of the model fits vary with the inclusion of the kinematic correction, the agreement between model and observation is qualitatively unchanged, and the results are discussed together below.

[32] Figure 6 (top) shows $S=\phi f_{C M E}$ and $L=\chi$ with best fit parameters of $\phi=3.3 \times 10^{11} \mathrm{~Wb} / \mathrm{CME}$ and $\chi=1.7 \times$ $10^{13} \mathrm{~Wb} \mathrm{CR}^{-1}$. These parameters are summarized in Table 1 and translate to loss from pinching balancing flux added by CMEs when $f_{C M E} \sim 50 \mathrm{CR}^{-1}\left(\approx 1.9 \mathrm{~d}^{-1}\right)$. The model variation in open solar flux is therefore very simple: During 19961998 , the CME rate is below this value and $\Phi_{O S}$ declines. For 1998-2003, $f_{C M E}$ exceeds this threshold and $\Phi_{O S}$ rises. Finally, around 2006, the CME rate drops below $1.9 \mathrm{~d}^{-1}$ and $\Phi_{O S}$ drops. The magnitude of the $\Phi_{O S}$ variation is approximately matched by the model, but it is clearly out of phase with that observed. Furthermore, a constant loss term produces a single solar maximum peak in $\Phi_{O S}$, rather than the asymmetric double peak which is observed.

[33] Figure 6 (middle) shows $S=\phi f_{C M E}$ and $L=\chi \Phi_{C H}(t)$, where $\Phi_{C H}(t)$ is the instantaneous closed heliospheric flux, essentially the model source term. Best fit parameters of $\phi=$ $4.4 \times 10^{11} \mathrm{~Wb} / \mathrm{CME}$ and $\chi=3.6 \times 10^{-2} \mathrm{CR}^{-1}$. Thus $\sim 4 \%$ of the closed heliospheric flux is lost per Carrington rotation. The model variation in $\Phi_{O S}$ is in good agreement with the overall magnitude and phase of the observed variation, however, the asymmetry about the Gnevyshev gap is absent. Furthermore, the decline in $\Phi_{O S}$ during this recent minimum is underestimated.

[34] Figure 6 (bottom) shows $S=\phi f_{C M E}$ and $L=\chi I_{I N C}(t)$ $\Phi_{O S}(t)$ using best fit parameters of $\phi=2 \times 10^{12} \mathrm{~Wb} / \mathrm{CME}$ and $\chi=4 \mathrm{CR}^{-1}$. Thus the rate of open solar flux loss is proportional to the current open solar flux and the fraction of the HCS which is inclined to the solar rotation direction. As $I_{I N C}$ is of order $10^{-2}$ and $\Phi_{O S}$ is of order $1 \times 10^{15}$, this is a very similar ratio of source to loss rates as the previous two models. The overall phase and amplitude of the variation in $\Phi_{O S}$ are well matched by this scheme. This model predicts a large drop in open solar flux around the year 2000, the time of the polarity reversal, which is not observed.

\subsection{Sunspot Number-Dependent $\Phi_{o S}$ Source Term}

[35] Next we consider an open solar flux source term which varies as the sunspot number, such that $S=\phi R$, where $\phi$ represents the average rate of flux injection per sunspot and $R$ is sunspot number. Note that, unlike using the CME rate, this can be applied to the whole sequence of data for which open solar flux values can be computed. Data are not used before 1976 because the solar wind flow data are not sufficiently continuous to allow the kinematic correction to be made [Lockwood et al., 2009b] and Wilcox magnetograms are unavailable to give an inclination of the HCS tilt. 


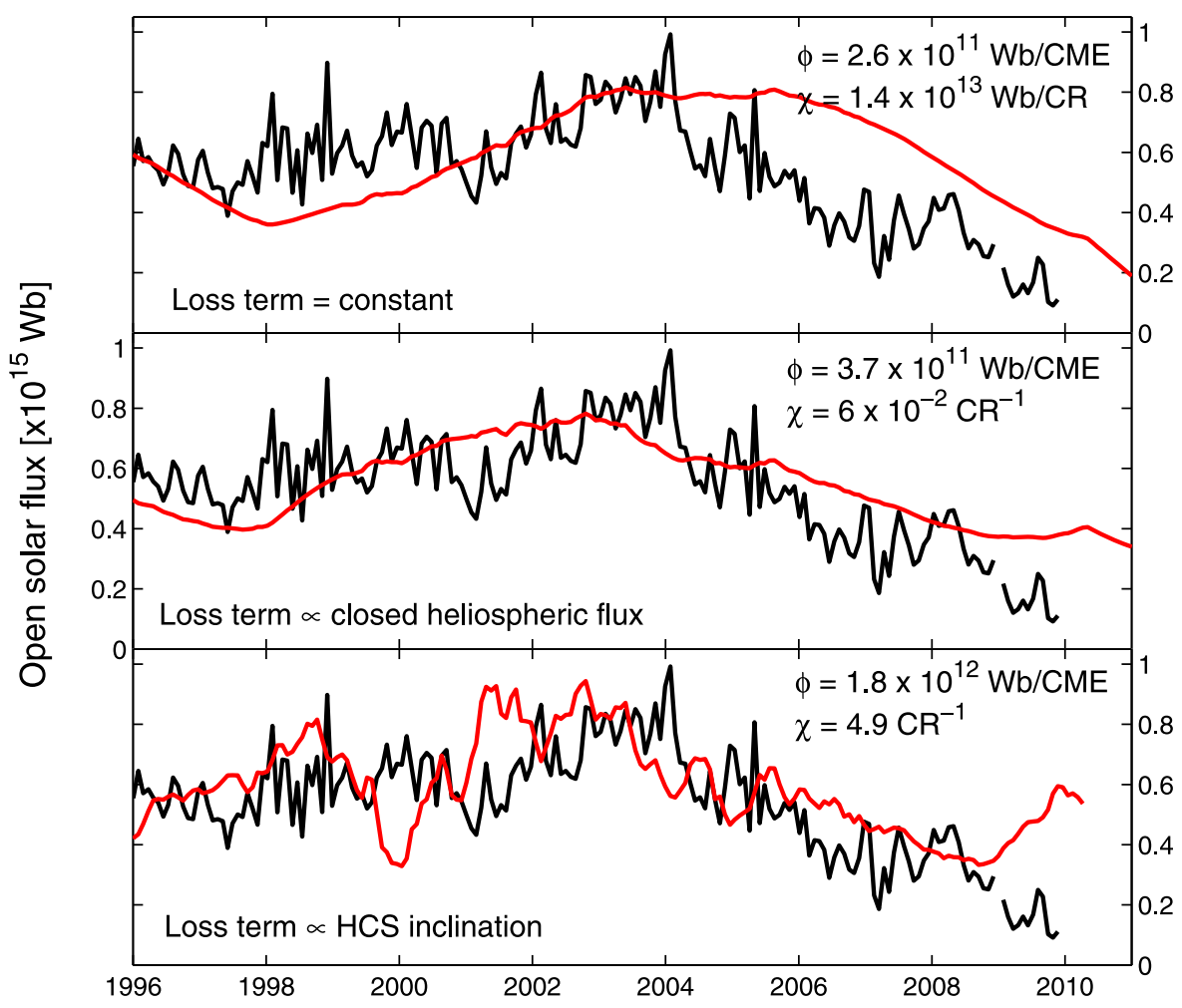

Figure 7. Same as Figure 6 but for open solar flux inferred from 1 AU measurements of hourly means $B_{R}$ kinematically corrected to the source surface.

[36] Figures 8 and 9 show model comparisons to open solar flux from hourly means of 1 AU observations of $B_{R}$ and $B_{R}$ kinematically corrected to the source surface, respectively.

[37] Figure 8 (top) shows $S=\phi R$ and $L=\chi$ with best fit parameters of $\phi=1.4 \times 10^{11} \mathrm{~Wb} \mathrm{R}^{-1} C R^{-1}$ and $\chi=1.1 \times$ $10^{13} \mathrm{~Wb} \mathrm{CR}^{-1}$. These values are approximately equal to each sunspot contributing one CME per Carrington rotation and the pinching process removing 100 CMEs worth of flux per CR [Lynch et al., 2006]. The constant loss rate means this model has similarities to the long- timescale loss terms of Schwadron et al. [2010], Krivova et al. [2007], Vieira and Solanki [2010], and Connick et al. [2010]. The general envelope of the solar cycle variation is well matched, with some of the cycle-to-cycle amplitude variations captured in a qualitative sense. There is, however, a slight phase offset, with the minima in open solar flux being estimated approximately a year later than observed. The Gnevyshev gap is also entirely absent from the model, as is any $\Phi_{O S}$ asymmetry about solar maximum. The constant removal of $\Phi_{O S}$ during the recent extended period of low sunspot number means the decline in $\Phi_{O S}$ during the recent solar minimum is well captured by this model [Schwadron et al., 2010].

[38] Figure 8 (middle) shows $S=\phi R$ and $L=\chi \Phi_{C H}(t)$, where $\Phi_{C H}(t)$ is again the instantaneous closed heliospheric flux. Best fit parameters of $\phi=6 \times 10^{11} \mathrm{~Wb}^{-1} C R^{-1}$ and $\chi=$ $1.5 \times 10^{-2} \mathrm{CR}^{-1}$ are used. This approach is similar to that of Owens and Crooker [2006] and comparable to Krivova et al. [2007] and Vieira and Solanki [2010] without the longtimescale ephemeral variation. The open solar flux variation is approximately in phase with that observed and the relative amplitudes of the cycles are in general agreement. While $\Phi_{O S}$ does plateau around solar maximum, there is still no reproduction of the Gnevyshev gap nor of the $\Phi_{O S}$ asymmetry about the gap. Note that in this scheme, the recent $\Phi_{O S}$ decline is slightly underpredicted, as the $L$ effectively falls off with the $S$.

Table 1. Source and Loss Terms for the Model Fits to Open Solar Flux Computed From Observed $\left|B_{R}\right|^{\mathrm{a}}$

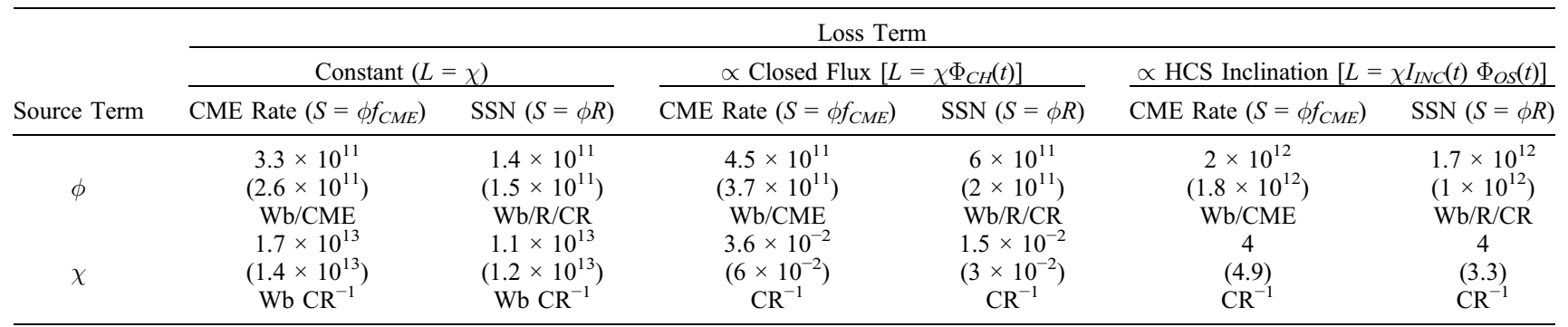

${ }^{\mathrm{a}}$ Fit parameters to kinematically corrected $\left|B_{R}\right|$ are shown in brackets. 


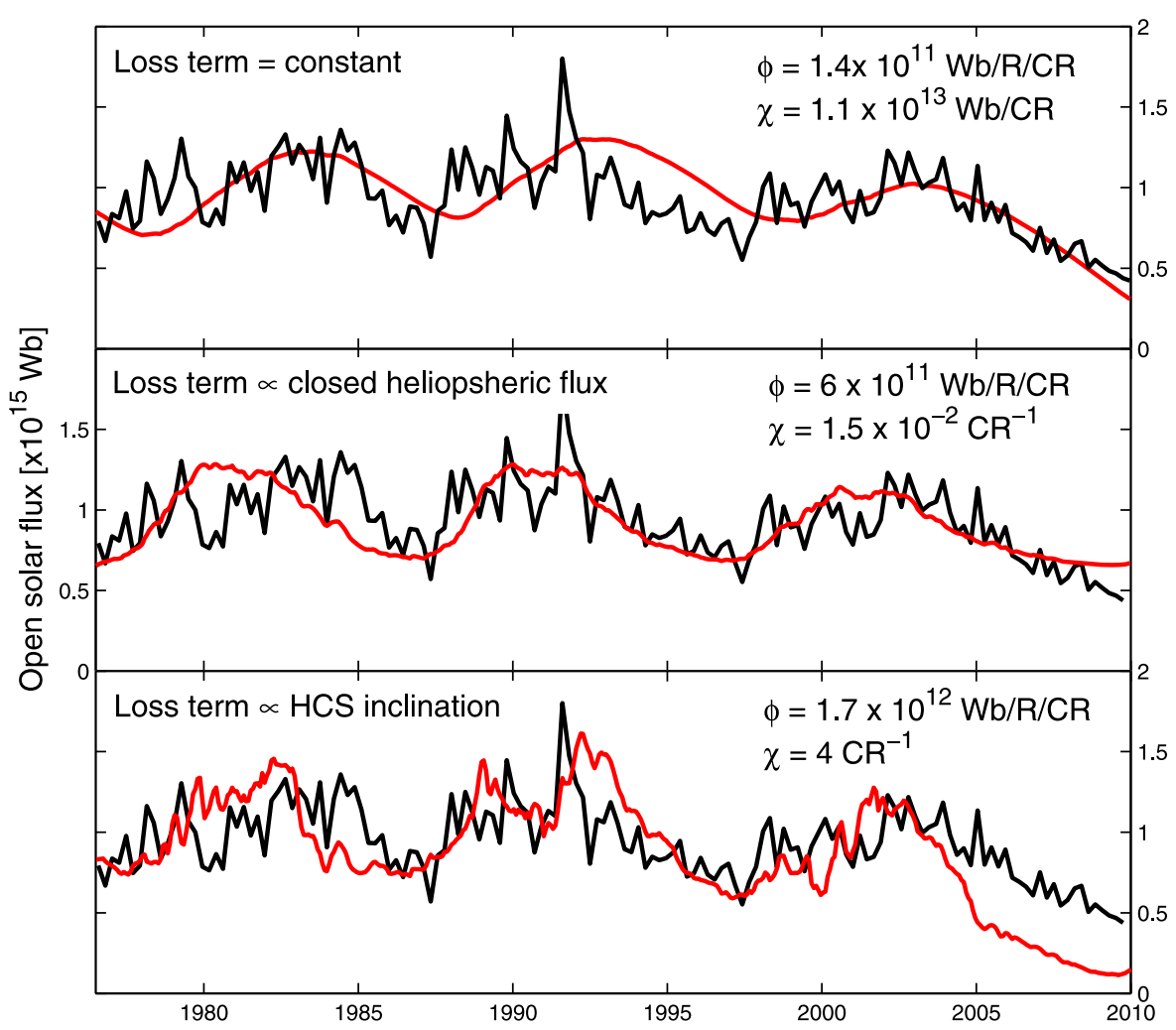

Figure 8. Modeling open solar flux using the observed sunspot number. Black lines show three Carrington rotation averages of the open solar flux computed from $1 \mathrm{AU}$ observations of $B_{R}$. The red lines show model reconstructions using the sunspot number as the source term. From top to bottom, the panels show open solar flux loss at a constant rate, at a rate proportional to the amount of closed heliospheric flux, and at a rate proportional the local HCS inclination.

[39] Figure 8 (bottom) shows $S=\phi R$ and $L=\chi I_{I N C}(t)$ $\Phi_{O S}(t)$ using best fit parameters of $\phi=1.7 \times 10^{12} \mathrm{~Wb} R^{-1}$ $C R^{-1}$ and $\chi=4 \mathrm{CR}^{-1}$. The overall phase and amplitude of the variation in $\Phi_{O S}$ are well matched by this scheme. The Gnevyshev gap is present for both cycles 22 and 23 (although slightly early in the case of cycle 23), as is the correct asymmetry in $\Phi_{O S}$ about that point. The decline in $\Phi_{O S}$ during the last three solar minima is clearly present, however, this model overpredicts the loss of open solar flux in the declining phase leading to the most recent minimum. Figure 9 shows that the model and observed $\Phi_{O S}$ are in better agreement when the kinematic corrected is applied to the $1 \mathrm{AU} B_{R}$ measurements. These findings are discussed in section 5 .

\section{Discussion}

[40] In this study we combined in situ heliospheric observations, remote solar photospheric observations and potential field source surface modeling of the corona to investigate how open solar flux is lost over the solar cycle. We assumed that the source of open solar flux, i.e., closed loops added to the heliosphere, proceeds at a rate proportional to either the sunspot number $(\mathrm{SSN})$ or the CME rate.

[41] We first used CME rates over the period 1996-present (i.e., SOHO and STEREO coronagraph observations) as the open solar flux source term. Three possibilities for the open solar flux loss rate were considered: A constant rate, a rate proportional to the amount of closed heliospheric flux and a rate proportional to the local inclination of the heliospheric current sheet (HCS). The assumption of constant open solar flux loss yields an open solar flux variation out of phase with that observed. Assuming open solar flux loss proportional to the closed heliospheric flux the correct phase is obtained, though the asymmetry about solar maximum is absent. The best match to observations is obtained assuming open flux loss at a rate modulated by the local inclination of the HCS. This model, however, predicts a large drop in open solar flux during 2000, the time of the global magnetic polarity reversal, which is not observed. There are a number of possible reasons for this discrepancy. It is possible that the potential field estimates of HCS inclination are not accurate during this period of rapid coronal evolution, or that CMEs are more difficult to identify when the corona is so dynamic. Alternatively, the relations between HCS inclination and open solar flux loss or between CME rate and flux injection could be substantially different during the polarity reversal than the rest of the solar cycle.

[42] It is obviously desirable to test whether this model of open solar flux holds for previous solar cycles, in particular, whether it is able to reproduce the observed cycle-to-cycle variability. As intercalibrated CME rates are not available before 1996, the period of analysis was extended using 


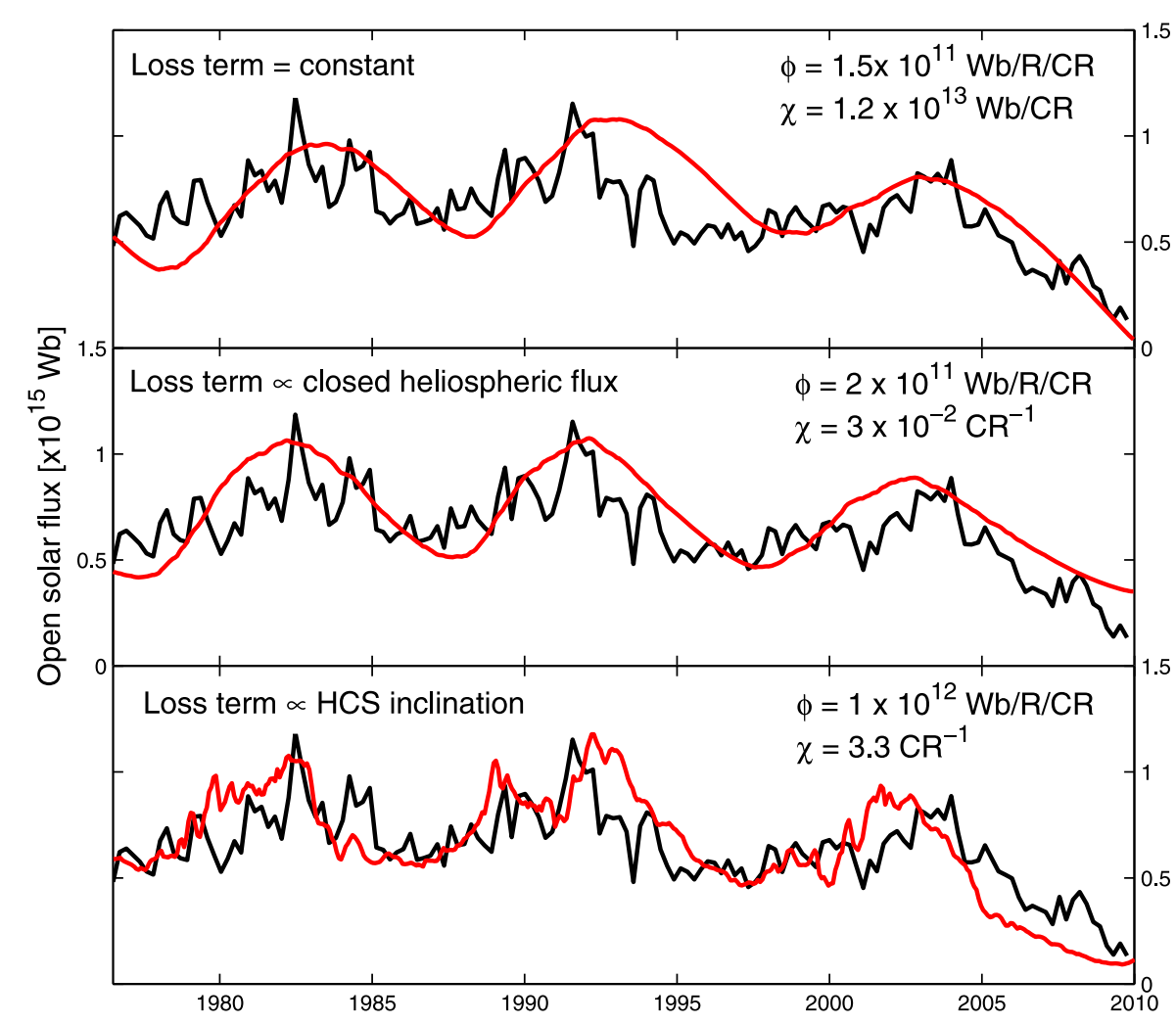

Figure 9. Same as Figure 8 but for open solar flux inferred from 1 AU measurements of $B_{R}$ kinematically corrected to the source surface.

sunspot number as a proxy for CME rate, as SSN is known to correlate with the coronal mass ejection (CME) rate. Assumptions of constant flux loss and loss proportional to the closed heliospheric flux are unable to reproduce two major features of the solar cycle open solar flux variation, namely the Gnevyshev gap, which is a drop in open solar flux at the peak of solar maximum, and the asymmetry in open solar flux about this point. The assumption of open solar flux loss modulated by the degree of HCS warping, however, does reproduce these features without any additional free parameters. This suggests that open solar flux is primarily lost at locations where the HCS is inclined to the solar rotation direction, as white light observations of the corona have previously suggested [Sheeley and Wang, 2001]. We note, however, that CME rate shows many features observed in the open solar flux time series. Thus the use of CME rate, rather than sunspot number, as the open solar flux source term may result in a weaker dependence on HCS warping. Unfortunately, the lack of multiple solar cycles of consistent CME rate observations means it is not currently possible to test this idea.

[43] Our HCS inclination model overestimates open solar flux loss during the decline into the most recent solar minimum, though the magnitude of the overestimate is reduced when the $1 \mathrm{AU}$ observations of open solar flux are corrected for kinematic amplification between the source surface and 1 AU. The overestimate is likely the result of the simplified source term, which assumes open solar flux is created at a rate proportional to sunspot number. As sunspot number approaches zero, this may not be valid. Indeed, although SSN reached zero at the end of cycle 23 , the CME rate

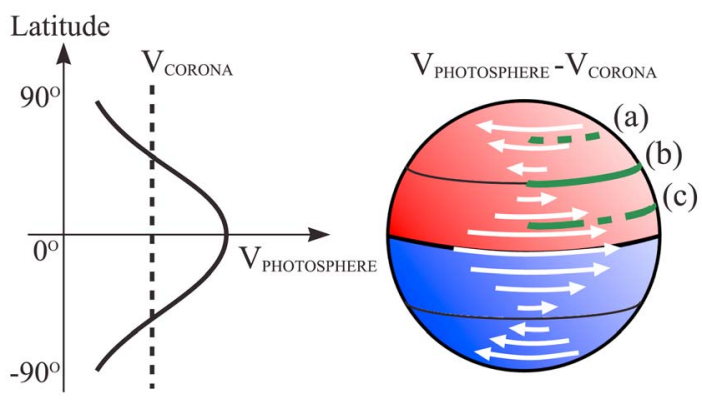

(a)

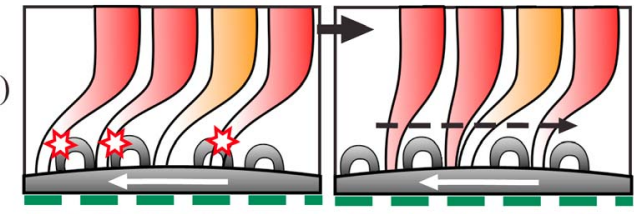

(b)

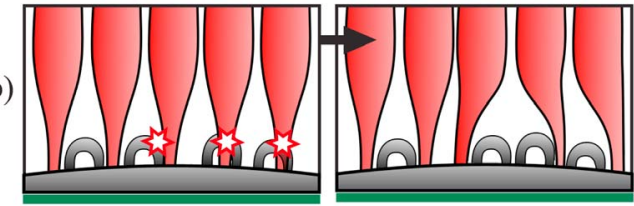

(c)

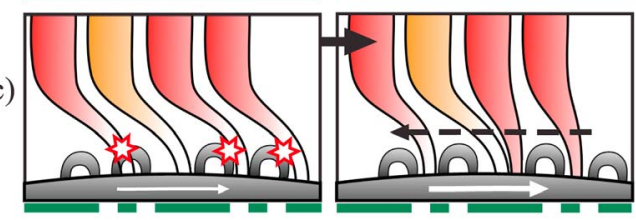

Figure 10. The transport of open flux resulting from differential rotation of the photosphere. See text for a detailed description. 


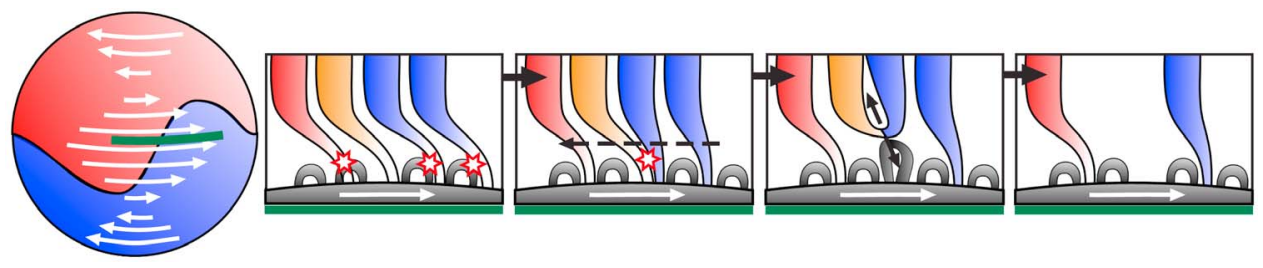

Figure 11. Open flux transport in the presence of an inclined current sheet. See text for a detailed description.

remained around $0.5 \mathrm{~d}^{-1}$, similar to the previous minimum (see also Figure 3) [Owens et al., 2008c]. Furthermore, during extended periods of zero sunspots such as the Maunder Minimum, the Sun is likely to have continued its polarity cycle, requiring a continued injection of open solar flux despite the lack of sunspots [Lockwood, 2006].

[44] Determination of the mechanism by which HCS inclination modulates open solar flux will require further modeling and observational efforts. It seems likely, however, that the transport of open flux by differential rotation [e.g., Fisk et al., 1999; Sheeley et al., 2001; Wang and Sheeley, 2004], may play a central role. Figure 10 shows a sketch of this process. The rotation speed of the photosphere varies systematically with latitude, peaking at the equator, while the corona rotates rigidly at a speed matched by the midlatitude photosphere. At this latitude (Figure 10b) in the bottom diagrams, open flux reconnects with emerging closed loops, shown in black, to randomly move open flux behind and ahead of the flux moving at the rotation speed, resulting in no net motion. At higher latitudes (Figure 10a), flux tubes become longitudinally sheared as the corona moves ahead of the photosphere. As a result of this relative motion, reconnection between open flux and small-scale closed loops has a systematic preference to reduce shear, with open flux foot points "slipping" ahead of the photospheric magnetic field. The process is quasi-random, meaning flux tubes bunch at longitudes where reconnection has yet to occur, shown in orange. At lower latitudes (Figure 10c), open flux is sheared, and hence moves, in the opposite sense.

[45] Figure 11 shows the pattern of open flux transport in the presence of an inclined heliospheric current sheet. In this scenario, the "slipping" of flux tubes will intermittently force together open flux of opposite polarity [see also Lionello et al., 2006] at rates that are highest where the local tilt is greatest. Reconnection between such flux tubes results in a completely disconnected loop which will propagate out with the solar wind and hence reduce the open solar flux. The sunward portion of the reconnected field lines move back toward the photosphere, resulting in coronal inflows observed at the location of a tilted current sheet [Sheeley and Wang, 2001]. It thus seems reasonable to assume that the rate at which pinching proceeds may be determined by HCS inclination. In this view, open solar flux will continue to be shed until the HCS is completely rotation aligned. This interplay between poloidal and toroidal solar magnetic flux systems may have implications for solar dynamo models.

[46] It should be reiterated that both CME rate/sunspot number and HCS inclination, the open solar flux source and loss terms, respectively, are set by the photospheric flux distribution. Ultimately, open solar flux is determined purely by the internal processes which set the photospheric boundary condition. This study is concerned with changes to photosphere flux that can be communicated through the corona to the heliosphere, much in the same way that the HCS can be tilted by the addition and removal of closed flux to the heliosphere by CMEs [Owens et al., 2007]. Finally, we note that as HCS inclination is strongly affected by the size and location of sunspot groups, particularly at solar minimum, it may be possible to empirically characterize open solar flux on the basis of historical sunspot distribution records. This would be valuable for understanding the link between prehistoric cosmic ray records and solar irradiance for terrestrial climate modeling.

[47] Acknowledgments. N.C. is supported by NSF grant AGS0962645. We are grateful to the Space Physics Data Facility (SPDF) and National Space Science Data Center (NSSDC) for OMNI data and to T. Hoeksema of Stanford University for WSO magnetograms. M.O. thanks J. Luhmann of University of California, Berkeley for the use of the potential field source surface model.

[48] Philippa Browning thanks Duncan Mackay and another reviewer for their assistance in evaluating this manuscript.

\section{References}

Altschuler, M. A., and G. Newkirk Jr. (1969), Magnetic fields and the structure of the solar corona, Sol. Phys., 9, 131-149.

Arge, C. N., and V. J. Pizzo (2000), Improvement in the prediction of solar wind conditions using near-real time solar magnetic field updates, J. Geophys. Res., 105, 10,465-10,479.

Arge, C. N., E. Hildner, V. J. Pizzo, and J. W. Harvey (2002), Two solar cycles of nonincreasing magnetic flux, J. Geophys. Res., 107(A10), 1319, doi:10.1029/2001JA000503.

Cane, H. V., G. Wibberenz, I. G. Richardson, and T. T. von Rosenvinge (1999), Cosmic ray modulation and the solar magnetic field, Geophys. Res. Lett., 26, 565-568, doi:10.1029/1999GL900032.

Connick, D. E., C. Smith, and N. A. Schwadron (2010), Interplanetary magnetic flux depletion during protracted solar minima, Astrophys. J., $727,8$.

Crooker, N. U., J. T. Gosling, and S. W. Kahler (2002), Reducing heliospheric magnetic flux from coronal mass ejections without disconnection, J. Geophys. Res., 107(A2), 1028, doi:10.1029/2001JA000236.

Crooker, N. U., S. W. Kahler, J. T. Gosling, and R. P. Lepping (2008), Evidence in magnetic clouds for systematic open flux transport on the Sun, J. Geophys. Res., 113, A12107, doi:10.1029/2008JA013628.

Fisk, L. A., T. H. Zurbuchen, and N. A. Schwadron (1999), Coronal hole boundaries and their interaction with adjacent regions, Space Sci. Rev., 87, 43-54.

Gnevyshev, M. N. (1977), Essential features of the 11 year solar cycle, Sol. Phys., 51, 175-183.

Gopalswamy, N., S. Yashiro, G. Michalek, G. Stenborg, A. Vourlidas, S. Freeland, and R. Howard (2008), The SOHO/LASCO CME catalog, Earth Moon Planets, 104, 295-313.

Gosling, J. T., D. N. Baker, S. J. Bame, W. C. Feldman, and R. D. Zwickl (1987), Bidirectional solar wind electron heat flux events, J. Geophys. Res., 92, 8519-8535.

Jones, G. H., A. Balogh, and E. J. Smith (2003), Solar magnetic field reversal as seen at Ulysses, Geophys. Res. Lett., 30(19), 8028, doi:10.1029/ 2003GL017204 
Krivova, N. A., L. Balmaceda, and S. K. Solanki (2007), Reconstruction of solar total irradiance since 1700 from the surface magnetic flux, Astron. Astrophys., 467, 335-346, doi:10.1051/0004-6361:20066725.

Lionello, R., J. A. Linker, Z. Mikić, and P. Riley (2006), The latitudina excursion of coronal magnetic field lines in response to differential rotation: MHD simulations, Astrophys. J., 642, L69-L72, doi:10.1086/ 504289

Lockwood, M. (2006), What do cosmogenic isotopes tell us about past solar forcing of climate?, Space Sci. Rev., 125, 95-109, doi:10.1007/ s11214-006-9049-2.

Lockwood, M., and M. Owens (2009), The accuracy of using the Ulysses result of the spatial invariance of the radial heliospheric field to compute the open solar flux, Astrophys. J., 701, 964-973, doi:10.1088/0004637X/701/2/964.

Lockwood, M., R. J. Forsyth, A. Balogh, and D. J. McComas (2004), Open solar flux estimates from near-Earth measurements of the interplanetary magnetic field: Comparison of the first two perihelion passes of the Ulysses spacecraft, Ann. Geophys., 22, 1395-1405.

Lockwood, M., M. Owens, and A. P. Rouillard (2009a), Excess open solar magnetic flux from satellite data: 1 . Analysis of the third perihelion Ulysses pass, J. Geophys. Res., 114, A11103, doi:10.1029/2009JA014449.

Lockwood, M., M. Owens, and A. P. Rouillard (2009b), Excess open solar magnetic flux from satellite data: 2. A survey of kinematic effects, J. Geophys. Res., 114, A11104, doi:10.1029/2009JA014450.

Low, B. C. (2001), Coronal mass ejections, magnetic flux ropes, and solar magnetism, J. Geophys. Res., 106, 25,141-25,160.

Luhmann, J. G., J. T. Gosling, J. T. Hoeksmam, and X. Zhao (1998), The relationship between large-scale magnetic field evolution and corona mass ejections, J. Geophys. Res., 103, 6585-6593.

Luhmann, J. G., D. Larson, J. T. Hoeksama, X.-P. Zhao, C. N. Arge, and O. C. St. Cyr (1999), Connections between the slow solar wind, CMEs, and the helmet streamer belt inferred from coronal field models, in Solar Wind Nine: Proceedings of the Ninth International Solar Wind Conference, Nantucket, Massachusetts, October, 1998, edited by S. R. Habbal et al., pp. 725-728, Am. Inst. of Phys., Woodbury, N. Y.

Lynch, B. J., J. R. Gruesbeck, T. H. Zurbuchen, and S. K. Antiochos (2006), Solar cycle-dependent helicity transport by magnetic clouds, J. Geophys. Res., 110, A08107, doi:10.1029/2005JA011137.

McComas, D. J., J. T. Gosling, and J. L. Phillips (1992), Interplanetary magnetic flux: Measurement and balance, J. Geophys. Res., 97, 171-177.

Nash, A. G., N. R. Sheeley Jr., and Y.-M. Wang (1988), Mechanisms for the rigid rotation of coronal holes, Sol. Phys., 117, 359-389.

Olmedo, O., J. Zhang, H. Wechsler, A. Poland, and K. Borne (2008), Automatic detection and tracking of coronal mass ejections in coronagraph time series, Sol. Phys., 248, 485-499, doi:10.1007/s11207-007-9104-5.

Owens, M. J., and N. U. Crooker (2006), Coronal mass ejections and magnetic flux buildup in the heliosphere, J. Geophys. Res., 111, A10104, doi:10.1029/2006JA011641

Owens, M. J., and N. U. Crooker (2007), Reconciling the electron counterstreaming and dropout occurrence rates with the heliospheric flux budget, J. Geophys. Res., 112, A06106, doi:10.1029/2006JA012159.

Owens, M. J., C. N. Arge, H. E. Spence, and A. Pembroke (2005), An event-based approach to validating solar wind speed predictions: High speed enhancements in the Wang-Sheeley-Arge model, J. Geophys Res., 110, A12105, doi:10.1029/2005JA011343.

Owens, M. J., N. A. Schwadron, N. U. Crooker, W. J. Hughes, and H. E. Spence (2007), Role of coronal mass ejections in the heliospheric Hale cycle, Geophys. Res. Lett., 34, L06104, doi:10.1029/2006GL028795.

Owens, M. J., C. N. Arge, N. U. Crooker, N. A. Schwadron, and T. S Horbury (2008a), Estimating total heliospheric magnetic flux from singlepoint in situ measurements, J. Geophys. Res., 113, A12103, doi:10.1029/ 2008JA013677.

Owens, M. J., N. U. Crooker, and N. A. Schwadron (2008b), Suprathermal electron evolution in a Parker spiral magnetic field, J. Geophys. Res. 113, A11104, doi:10.1029/2008JA013294.

Owens, M. J., N. U. Crooker, N. A. Schwadron, T. S. Horbury, S. Yashiro, H. Xie, O. C. St Cyr, and N. Gopalswamy (2008c), Conservation of open solar magnetic flux and the floor in the heliospheric magnetic field, Geophys. Res. Lett., 32, L20108, doi:10.1029/2008GL035813.

Richardson, I. G., H. V. Cane, and E. W. Cliver (2002), Sources of geomagnetic activity during nearly three solar cycles (1972-2000), J. Geophys. Res., 107(A8), 1187, doi:10.1029/2001JA000504.
Riley, P. (2007), An alternative interpretation of the relationship between the inferred open solar flux and the interplanetary magnetic field, Astrophys. J., 667, L97-L100, doi:10.1086/522001.

Robbrecht, E., and D. Berghmans (2004), Automated recognition of coronal mass ejections (CMEs) in near-real-time data, Astron. Astrophys., 425, 1097-1106, doi:10.1051/0004-6361:20041302.

Schatten, K. H., J. M. Wilcox, and N. F. Ness (1969), A model of interplanetary and coronal magnetic fields, Sol. Phys., 9, 442-455.

Schüssler, M., and I. Baumann (2006), Modeling the Sun's open magnetic flux, Astron. Astrophys., 459, 945-953, doi:10.1051/0004-6361:20065871.

Schwadron, N. A., D. E. Connick, and C. Smith (2010), Magnetic flux balance in the heliosphere, Astrophys. J., 722, L132-L136, doi:10.1088/ 2041-8205/722/2/L132.

Sheeley, N. R., Jr., and Y.-M. Wang (2001), Coronal inflows and sector magnetism, Astrophys. J., 562, L107-L110, doi:10.1086/338104.

Sheeley, N. R., Jr., T. N. Knudson, and Y.-M. Wang (2001), Coronal inflows and the Sun's nonaxisymmetric open flux, Astrophys. J., 546 , L131-L135.

Smith, E. J., and A. Balogh (2003), Open magnetic flux: Variation with latitude and solar cycle, in Solar Wind Ten: Proceedings of the Tenth International Solar Wind Conference, Pisa, Italy, 17-21 June 2002, AIP Conf. Proc., vol. 679, edited by M. Velli, R. Bruno, and F. Malara, pp. 67-70, Am. Inst. of Phys., Melville, N. Y.

Solanki, S. K., M. Schüssler, and M. Fligge (2000), Evolution of the Sun's large-scale magnetic field since the Maunder minimum, Nature, 408 , 445-447, doi:10.1038/35044027.

St. Cyr., O. C., et al. (2000), Properties of coronal mass ejections: SOHO LASCO observations from January 1996 to June 1998, J. Geophys. Res. $105,18,169-18,185$

Vieira, L. E. A., and S. K. Solanki (2010), Evolution of the solar magnetic flux on time scales of years to millenia, Astron. Astrophys., 509, A100, doi:10.1051/0004-6361/200913276.

Wang, Y., and N. R. Sheeley Jr. (2004), Footpoint switching and the evolution of coronal holes, Astrophys. J., 612, 1196-1205, doi:10.1086/ 422711

Wang, Y., and N. R. Sheeley (2009), Understanding the geomagnetic precursor of the solar cycle, Astrophys. J., 694, L11-L15, doi:10.1088/0004637X/694/1/L11.

Wang, Y.-M., and N. R. Sheeley Jr. (1995), Solar implications of Ulysses interplanetary field measurements, Astrophys. J., 447, L143-L146, doi:10.1086/309578.

Wang, Y.-M., and N. R. Sheeley Jr. (2003), On the topological evolution of the coronal magnetic field during the solar cycle, Astrophys. J., 599, $1404-1417$

Wang, Y.-M., N. R. Sheeley, R. A. Howard, N. B. Rich, and P. L. Lamy (1999), Streamer disconnection events observed with the LASCO coronagraph, Geophys. Res. Lett., 26, 1349-1352, doi:10.1029/ 1999GL900177.

Webb, D. F., and R. A. Howard (1994), The solar cycle variation of coronal mass ejections and the solar wind mass flux, J. Geophys. Res., 99, 4201-4220.

Wilcox, J. M., and A. J. Hundhausen (1983), Comparison of heliospheric current sheet structure obtained from potential magnetic field computations and from observed polarization coronal brightness, J. Geophys Res., 88, 8095-8096, doi:10.1029/JA088iA10p08095.

Yashiro, S., N. Gopalswamy, G. Michalek, O. C. St Cyr, S. P. Plunkett, N. B Rich, and R. A. Howard (2004), A catalog of white light coronal mass ejections observed by the SOHO spacecraft, J. Geophys. Res., 109, A07105, doi:10.1029/2003JA010282.

Yashiro, S., G. Michalek, and N. Gopalswamy (2008), A comparison of coronal mass ejections identified by manual and automatic methods, Ann. Geophys., 26, 3103-3112, doi:10.5194/angeo-26-3103-2008.

Yeates, A. R., D. H. Mackay, A. A. van Ballegooijen, and J. A. Constable (2010), A nonpotential model for the Sun's open magnetic flux, 115, A09112, doi:10.1029/2010JA015611.

N. U. Crooker, Center for Space Physics, Boston University, Boston, MA 02215, USA.

M. Lockwood and M. J. Owens, Space Environment Physics Group, Department of Meteorology, University of Reading, Earley Gate, PO Box 243, Reading RG6 6BB, UK. (m.j.owens@reading.ac.uk) 